\title{
ECG SIGNAL ANALYSIS OF EARLY AND LATE RISERS: A POPULATION BASED CASE CONTROL STUDY
}

\author{
Muhammad Hassan ${ }^{\mathrm{a}}$, Sami Ur Rahman ${ }^{\mathrm{b}}$ and Zhou You ${ }^{\mathrm{a}}$ \\ ${ }^{a}$ College of Computer Science and Technology, Jilin University, China \\ ${ }^{b}$ Department of Computer Science and Information Technology, University of Malakand, Khyberpakhtunkhwa, Pakistan
}

\begin{abstract}
This paper investigates the effect of early rising on heart rhythm. We have analyzed the ECG signals of early (or Controls) and late (or Cases) risers. In the study we obtained ECG signals of two hundred healthy humans having age range between twenty and thirty-five years. The twenty features of ECG signals were analyzed in the experiment. The significant difference in the two groups was reported based on the two features of the ECG signals i.e. QRS and QTc. Cases were associated with the variations in QRS complex and QTc features of their ECG signals. The QRS complex abnormal values were $76 \%$ for cases and $31 \%$ for controls. Similarly, the QTc was also found abnormal with $75 \%$ for cases and 33\% for controls. The accumulative abnormal values of both QRS and QTc were found 75\% and 48\% for late and early risers respectively. There is a relative high occurrence of heart abnormalities in cases than controls. The probability of QRS and QTc abnormality is more for Cases as compared to Controls.
\end{abstract}

\section{KEYWORDS}

Electrocardiogram (ECG), Circadian Cycle, Heart Rhythm, Early Rising, Late Rising

\section{INTRODUCTION}

Heart is an amazing muscular organ in the human body and other animals that pumps blood continuously to the different organs using vessels (Venes). The heart related variations in energy in different states of body can be measured from ECG. Electrocardiogram (ECG) is a measure of the wave form of energy generated by the heart and represents an individual cardiac feature. ECG has been analyzed by different researchers for various diseases identification (Dubin, 2000, Golden Jr et al., 1973). ECG has been used for examining of many activities, behaviours and characteristics like sleep, loneliness, smoking and other lifestyle (Kesper et al., 2012, Devi et al., Hawkley et al., 2003, Sherwood et al., 1990). Sleep and Cardiovascular health are interconnected with each other. Cardiovascular health is also effected from many habitual activities like smoking (Devi et al.). ECG signal disorderness and differential activation are also associated with people spent much their time in loneliness (Hawkley et al., 2003). The habit of getting up in the morning varies from person to person. Getting up early in the morning has been advocated in all the times and many researchers have described its social, economic, and psychological benefits. In this paper, Psycho-physiological and behavioral effects on human heart is studied. For this purpose, the subjects are selected and classified into Cases and Controls. The ECG signals of both the Cases and Controls are investigated and analyzed with considering the total number of 19-features including (P, Q, R, S and T wave) as well as QRS(depolarization) and QTc(repolarization).

\footnotetext{
${ }^{I}$ Corresponding Author: Zhou You, College of Computer Science and Technology, Jilin University, China Email-Address: zyou@jlu.edu.cn
} 


\section{RELATED WORK}

Getting up early in the morning has been advocated in all the times and many researchers have described its social, economic, and psychological benefits (Franklin, 1855). They have investigated the positive effects of early rising on social, academic and professional life. In the study of correlation of morningness-eveningness, it has been investigated that those students who wakeup early in the morning have shown significant academic outcomes and achievements than the late risers (Randler and Frech, 2006, Laino, 2008). The performance of morning-type (MT) students is better in school as compared to evening-type (ET) students (Den, 2000). A study conducted in Texas University(Laino, 2008) shows that the early morning risers have received high score in their GPAs as compared to the late risers. It has also been investigated that the student's biological rhythm is varied with sleepiness and it affects performance during daytime in school schedule (Andrade and Menna-Barreto, 1996). Harvard biologist, Christoph Randler revealed that early risers are more proactive(Randler, 2009). Randler's research also discovered that "morning people" are more likely to anticipate problems which leads to more success in the business world. It has also been explored that early risers exhibit character traits like satisfaction, optimism, being agreeable and consciousness (Ravi, 2015). On the other hand, night owls are associated with creativity and intelligence while they are more likely to exhibit traits like pessimism, depression, and being neurotic. Benjamin Franklin appreciated to go early to bed and wakeup early in the morning (Franklin, 1855). In the study of sleep and biological rhythm, it is suggested that variation in sleep-time influences hippocampus volume (Kuperczko et al., 2015). Hippocampus is a part of the human brain located under the Cerebral-Cortex and its volume size is related to short and long term memory (Seifert, 1983). Hippocampus is found significantly smaller in volume for the students with late wakeup time as well as for shorter and longer sleepers. According to the sleep experts, if you go to bed early and get up early in the morning, your body will be more in tune with the earth's circadian rhythms (Randler, 2009).Circadian cycles (circadian rhythm) are the physical, mental and behavioural changes in the human body that occur during the 24-hour cycle. In addition, it has also been reported that an exposure to the light in the morning is effective for patients with depression and seasonal effective disorder (Goel et al., Terman et al., 1989). Circadian rhythm is considered effective on cardiovascular system and its pattern associated with cardiac related diseases like myocardial ischemia and acute myocardial infarct, and sudden cardiac death (Portaluppi et al.). The day-night patterns are significantly associated with atrial arrhythmias-premature beats, tachycardia, atrial fibrillation, and flutter. There is a strong evidence that cardiovascular system have shown variations with the intrinsic-circadian-clock and causes many cardiovascular diseases including arrhythmia and temporal trends(Chen, 2015). A review is also carried out regarding of human behaviours and their association with daily life activities both in ancient and modern perspectives (Rahman and Hassan, 2014, Hassan et al., 2016). In this study, the source population for study members involved people having variation in their rising habits. The objective is to determine the strength of association between various heart abnormalities in late risers.

\section{METHODOLOGY}

\subsection{Selection of Cases and Controls}

The case-control study conducted on participants having age between twenty and thirty-five years, from August 2016 to November 2016. The participants are healthy and have not any history of heart diseases. The sources population are all those participants who have variations in their occupations and activities. The ECG signals are obtained during 9:00 am-12:00 pm. The participants are categorized such that, in which 80 participants from cases and 120 participants from controls are included in the given population. For Cases identification, all participants admitted to cardiology ward. Cases are eligible if they have characteristics symptoms plus electrocardiogram changes indicative of heart abnormalities. The controls are those participants who have not experienced any heart diseases. They found normal in their ECG recordings. Similarly, the age and sex-matched controls found identical to those described for cases, with additional criterion that control have no previous diseases. About 58\% controls are selected as hospital based and $42 \%$ are community-based or non-cardiac ward controls. 


\subsection{Study Questionnaires}

Structured questionnaires were administered to ask case-participants and controls about locality, age, and smoking history, current and previous medication. Similarly, physical examinations were undertaken in the same manner in cases and controls. Information about demographic factors, socioeconomic status (education, income), lifestyle (smoking, leisure time and physical activity), personal and family history of cardiovascular disease, behaviours (early and late rising) and risk factors (hypertension, diabetes mellitus) were obtained. Psychosocial factors (depression, locus of control, perceived stress, and life events) were systematically recorded. Following steps were taken to perform the ECG acquisition, features extraction and their analysis for any chances of diseases. In addition, information was collected regarding diagnosis of general health conditions, including asthma, lung disease (defined as emphysema, chronic lung problems and/or chronic bronchitis), heart disease (defined as heart problems from birth, rheumatic heart disease, angina, heart attack, and/or heart failure excluding hypertension), diabetes (type I or II), other metabolic disorders, kidney disease (defined as kidney transplant, renal failure, and/or dialysis), liver disease, blood disorders (defined as sickle cell disease, thalassemia, or hemoglobin problems), mental health diagnoses, neurologic conditions (defined as conditions that involve muscles, nerves, or the brain), immune suppression (defined as cancer, HIV infection, or immunosuppressive medication), and obstructive sleep apnea.

\subsection{ECG Acquisition}

Before taking ECG of an individual, five to ten minutes were given to each subject for relaxation. In the experiments, ECG was taken from Cardio-Care2000 Bionet machine (Bionet, 2015). This machine is facilitated with 12-channel leads to find accurate heart's electro-magnetic field. During experiments, every subject was guided about their position and orientation. The machine enabled us to extract ECG print out in 3-Chanels, 6-Chanels and 12-Chanels as well as in soft form in the computer with 10 and 60 second duration. The EKG-Plus II software (Bionet, 2015) was used on PC to visualize the ECG in different formats.

\subsection{Features Extraction}

We used nineteen features for each subject's ECG. These features are heart beats variation (PBS), PR interval, QRS duration and amplitude, QT/QTc, P-axis, amplitude and duration, T and R axis, T amplitudes and durations, Q amplitudes and durations and ST amplitude (Hassan et al.). The peak values are computed with setting-up a threshold value. The identification of the peak ( $\mathrm{R}$ locations) values are further used as a base to find more features including R-amplitude (highness) and duration (interval). Similarly, the skewness is measured by calculating the orientation of R-peaks. The RR interval is equal to one beat which is further multiplied with 60 seconds and it produces one beat per minute. Furthermore, the QRS-complex is the combination of Q, R and S wave (Figure 1). It is the most complicated and important part of ECG signal. The combination of all these points generates the QRS complex. The angles of $\mathrm{P}, \mathrm{R}$ and $\mathrm{T}$ (skewness of $\mathrm{P}, \mathrm{R}$ and T) wave along the axis are calculated as depicted in Figure 1 (Singh and Singh, 2011).

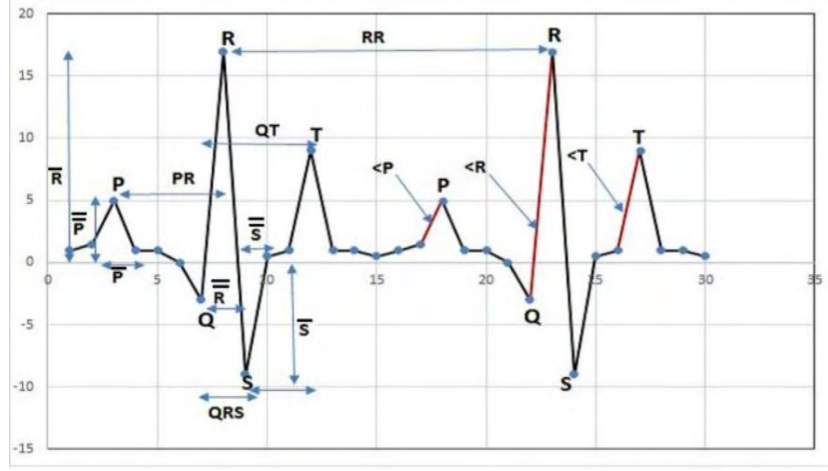

Figure 1. ECG signal with its intervals (QT, PR, $\bar{P}, \mathrm{QRS}, \overline{\bar{R}}, \overline{\bar{S}}$, $\mathrm{ST})$, amplitude $(\bar{R}, \overline{\bar{P}}, \bar{S}, \bar{Q})$ and Angle $(<\mathrm{P},<\mathrm{R},<\mathrm{T})$

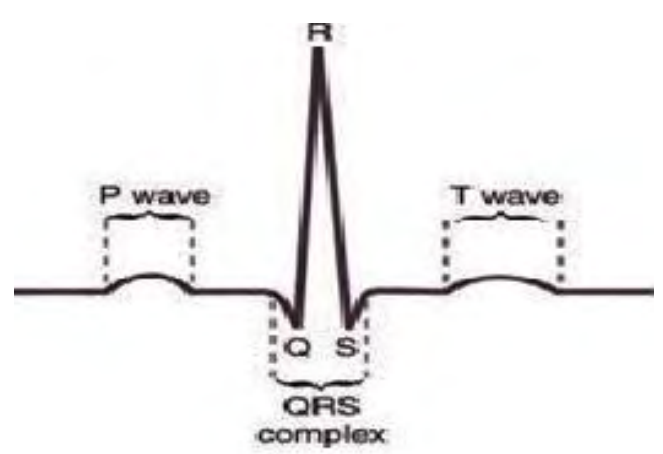

Figure 2. QRS Complex with Start and End Points 


\section{RESULTS ANALYSIS AND DISCUSSION}

In the result analysis and discussion, we describe the morphology of 1) QRS-Complex and 2) QT/QTc as well as their 3) statistical analysis. 1) QRS-Complex is the combination of three graphical deflection points (Q, R and S) in normal ECG (Trahanias, 1993). It is the central and most obvious part of ECG signal which indicates depolarization. The QRS complex starts with Q wave and ends with $\mathrm{S}$ wave (Figure 1). Normal duration and range of QRS complex is 0.06-0.01 seconds or 1.5 to 3 boxes (Becker, 2006) for male subjects. The normal duration of QRS complex is about 0.06-0.01 seconds, where variations in its normal range cause many types of abnormalities. A prolonged QRS complex indicates abnormalities in LVEF (Murkofsky et al., 1998) as well as increased LV ESCs and LV EDCs (Murkofsky et al., 1998). Similarly QRS changes also cause Myocardial Infarction, LVH and Anterior (Wagner et al., 1982). In addition to these abnormalities, exceeding the normal duration of QRS also causes diseases like WPWS, Ventricular-Tachycardia and LBBB (Chung et al., 1965). 2) In the QT/QTc Morphology, QT-interval is the time from the start of QRS-wave to the end of the T-wave. It represents repolarisation and depolarization of ventricles in the heart. The length of QT has inverse relation with the heart rate. The duration of QT decreases at faster heart rate and increases at slower heart rate. QTc is the corrected value to estimate the QT value over different heart rate to improve an accurate detection of arrhythmias. Normal range of QTc for men is 350-450 ms and 350-460 ms for women. Abnormal prolonged QT is associated with risk factor of ventricular arrhythmia where short QT interval is related with ventricular fibrillation, paroxysmal and sudden cardiac death. Different types of syndromes such as Hypokalaemia and Hypomagnesaemia have prolong QTc (Ponte et al., 2010). 3) In the statistical analysis, the proportions of characteristics among case-patients and controls were compared, as shown in Table 1. The comparison and evaluation of cases and controls are also shown in Table 2 and Table 3 with significant $\mathrm{P}$ value. In Table 1, QRS and QTc were considered from the ECG signal of cases and controls. The cases exposed to risk factor for QRS and QTc were 76\% and 75\% respectively. On the other hand, for controls, it is $31 \%$ and 33\% for QRS and QTc respectively. Similarly, further analysis of Odd Ratio (OR) can be read in Interpretation of Odd Ratio. The odds ratio is interpreted the same way as other ratio measures (risk ratio, rate ratio, etc.). The OR is 6.8 (more occurrences of QRS abnormal) and 1.5 based on QRS and QTc. A prolonged QRS complex indicates abnormalities in LVEF, LVESCs, LV EDCs (Murkofsky et al., 1998). Similarly, the OR for QTc also found greater than one (1), which indicates a high-risk factor of heart abnormalities in the cases. Abnormal prolonged and short QT is associated with risk factor of ventricular arrhythmia and ventricular fibrillation, paroxysmal, sudden cardiac death respectively while prolong QTc is associated with Hypokalaemia and Hypothermia (Ponte et al., 2010).

Table 1. Case controls study of early and late risers

\begin{tabular}{lllll}
\hline & Cases & \multicolumn{3}{c}{ Controls } \\
\hline & QRS & QTc & QRS & QTc \\
Exposed (late risers) & 63 & 60 & 37 & 40 \\
Non-Exposed (Early risers) & 20 & 20 & 80 & 80 \\
Total & 83 & 80 & 117 & 120 \\
\hline \%Exposed & $63 / 83=76 \%$ & $60 / 80=75 \%$ & $37 / 117=31 \%$ & $40 / 120=33 \%$ \\
\hline Interpretation of Odd Ratios: & & & \\
Odd Ratio=1 No Association & Odd Ratio $>\mathbf{1}$ & & Risk Factor | Odd Ratio<1 & Protective Factor
\end{tabular}

Odds ratio with respect to $\mathrm{QRS}=(63 \times 80) /(20 \times 37)=6.8>1$

Odds ratio with respect to $\mathrm{QTc}=(60 \times 80) /(80 \times 40)=1.5>1$

The OR values $(6.8,1.5)$ are greater than one (1) with respect to QRS complex and QTc, which indicates a high-risk factor of heart diseases in later risers.

Significance of the study: In order to determine whether the concerned features in the two groups (Cases and Controls) are showing significant variation or not, we have calculated ANOVA(ANOVA (F $(1,49)$ $=10.875, \mathrm{p}<0.00001)$ ) is for QRS features which is significant. Similarly, the ANOVA for QTc is $($ ANOVA $(\mathrm{F}(1,49)=13008.139, \mathrm{p}<0.00001)$ which is also significant. Two variables have been declared, 1) assignment of two values, 1 and 2 to Controls and Cases respectively. 2) Storing the actual values of the QRS and QTc. The ANOVA for QRS and QTc have been statistically generated as shown in Table 2-3 respectively. 
Table 2. Statistical Analysis of P value for QRS complex

\begin{tabular}{llllll}
\hline VAR00001 & Sum of Squares & Df & Means Square & F & Siq. \\
\hline Between Groups & SST $=15.000$ & $\mathrm{r}-1=12$ & MST $=1.250$ & F $=$ MST/MSE $=10.875$ & 0.000 \\
Within Groups & SSE $=10.000$ & $\mathrm{n}-\mathrm{r}=87$ & MSE $=0.115$ & & \\
Total & $\mathrm{SS}=25.000$ & $\mathrm{n}-1=99$ & & & \\
\hline
\end{tabular}

Table 3. Statistical Analysis of P value for QTc

\begin{tabular}{llllll}
\hline VAR00002 & Sum of Squares & Df & Means Square & F & Siq. \\
\hline Between Groups & 2419580.250 & 1 & 2419580.250 & $1.301 \mathrm{E} 4$ & 0.000 \\
Within Groups & 18228.500 & 98 & 186.005 & & \\
Total & 2437808.750 & 99 & & & \\
\hline
\end{tabular}

\section{CONCLUSION}

We investigated the effects of early and late rising on human heart using ECG signals. The significant difference found in the QRS complex and QTc interval among the participants of both groups. There are different diseases related to QRS complex abnormalities such as Left-Ventricular-Systolic-Dysfunctions and Left-Ventricular-Diastolic-Dysfunctions, Diastolic-Counts, Left Ventricular Hypertrophy, Wolf-Parkinson-White-Syndrome (WPWS), and Right-Left Buddle-Branch-Block. Moreover, QTc abnormalities are also associated with many diseases e.g. Hypokalaemia, Hypomagnesaemia, Hypothermia and Raised Intracranial Pressure. In the case-controls study, the Cases were found high risk of heart diseases compared to Controls. The statistical value of $\mathrm{P}$ and $\mathrm{OR}$ was significant which shows that the controls are healthier than cases.

\section{ACKNOWLEDGEMENT}

This research is supported by the National Natural Science Foundation of China (Grants No. 61772227, 61572227), the Science \& Technology Development Foundation of Jilin Province (Grants No. 20180201045GX) and the Science Foundation of Education Department of GuangdongProvince (Grants No. 2017KQNCX251, 2018XJCQSQ026) and the Social Science Foundation of Education Department of Jilin Province (Grants No. JJKH20181315SK)

\section{REFERENCES}

Andrade, M., M \& Menna-Barreto, L. (1996) Diurnal variation in oral temperature, sleepiness, and performance of high school girls. Biological Rhythm Research, 27, 336-342.

Becker, D. E. (2006) Fundamentals of electrocardiography interpretation. Anesthesia progress, 53, 53-64.

Bionet (2015) CardioCare 2000.

Chen, L. (2015) Recent advances in circadian rhythms in cardiovascular system. Frontiers in pharmacology, 6.

Chung, K.-Y., Walsh, T. J. \& Massie, E. (1965) Wolff-Parkinson-White syndrome. American heart journal, 69, 116-133.

Den, W. V. (2000) Time in bed, quality of sleep and school functioning of children. Journal of sleep research, 9, 145-153.

Devi, M. R., Arvind, T. \& Kumar, P. S. ECG Changes in Smokers and Non Smokers-A Comparative Study. Journal of clinical and diagnostic research: JCDR, 7, 824.

Dubin, D. (2000) Rapid interpretation of EKG's: an interactive course, Florida, United States, Cover Publishing Company.

Franklin, B. (1855) Early to Bed, and Early to Rise, Makes a Man Healthy, Wealthy, and Wise, Or, Early Rising, a Natural, Social, and Religious Duty, Theclassics US.

Goel, N., Basner, M., Rao, H. \& Dinges, D. F. Circadian rhythms, sleep deprivation, and human performance. Progress in molecular biology and translational science, 119, 155. 
Golden Jr, D. P., Wolthuis, R. A. \& Hoffler, G. (1973) A spectral analysis of the normal resting electrocardiogram. IEEE Transactions on Biomedical Engineering, 366-372.

Hassan, M., Rahman, S. U., Alam, F., Ali, Z. \& Alam, S. Effect of Rising Habit on the Human Health Using ECG signals. Journal of Postgraduate Medical Institute (Peshawar-Pakistan), 30.

Hassan, M., Rahman, S. U. \& Rahman, A. U. (2016) Heart's Role beyond a Blood Pumping Machine: A Literature Review in Modern Perspective.

J. Appl. Environ. Biol. Sci, 1, 197-210.

Hawkley, L. C., Burleson, M. H., Berntson, G. G. \& Cacioppo, J. T. (2003) Loneliness in everyday life: cardiovascular activity, psychosocial context, and health behaviors. Journal of personality and social psychology, 85, 105.

Kesper, K., Canisius, S., Penzel, T., Ploch, T. \& Cassel, W. (2012) ECG signal analysis for the assessment of sleep-disordered breathing and sleep pattern. Medical \& biological engineering \& computing, 50, 135-144.

Kuperczko, D., Perlaki, G., Faludi, B., Orsi, G., Altbacker, A., Kovacs, N., Doczi, T., Komoly, S., Schwarcz, A. \& Clemens, Z. (2015) Late bedtime is associated with decreased hippocampal volume in young healthy subjects. Sleep and Biological Rhythms, 13, 68-75.

Laino, C. (2008) Early Birds Get Better Grades. Sleep Disorders Health 1ed. sleep disorder center, Pomona Valley Hospital, California, WebMD News Archive.

Murkofsky, R. L., Dangas, G., Diamond, J. A., Mehta, D., Schaffer, A. \& Ambrose, J. A. (1998) A prolonged QRS duration on surface electrocardiogram is a specific indicator of left ventricular dysfunction. Journal of the American College of Cardiology, 32, 476-482.

Ponte, M. L., Keller, G. A. \& Girolamo, G. D. (2010) Mechanisms of drug induced QT interval prolongation. Current drug safety, 5, 44-53.

Portaluppi, F., Tiseo, R., Smolensky, M. H., Hermida, R. n. C., Ayala, D. E. \& Fabbian, F. Circadian rhythms and cardiovascular health. Sleep medicine reviews, 16, 151-166.

Rahman, S. U. \& Hassan, M. (2014) Heart's Role in the Human Body: A Literature Review. Journal of Applied Environmental and Biological Science, 4, 199-208.

Randler, C. (2009) Proactive People Are Morning People1. Journal of Applied Social Psychology, 39, 2787-2797.

Randler, C. \& Frech, D. (2006) Correlation between morningness-eveningness and final school leaving exams. Biological Rhythm Research, 37, 233-239.

Ravi, R. (2015) Why Even Night Owls Should Wake Up Early. Motivated Life. Southern California, Los Angeles, Ravi Raman Enterprises, LLC.

Seifert, W. (1983) Neurobiology of the Hippocampus, United States of America, Cambridge, Academic Press.

Sherwood, A., Dolan, C. A. \& Light, K. C. (1990) Hemodynamics of blood pressure responses during active and passive coping. Psychophysiology, 27, 656-668.

Singh, Y. N. \& Singh, S. K. (2011) Evaluation of electrocardiogram for biometric authentication.

Terman, M., Terman, J., Quitkin, F. \& McGrath, P. (1989) Bright light therapy for winter depression: A review of efficacy. Neuropsychopharmacology, 2, 1-22.

Trahanias, P. (1993) An approach to QRS complex detection using mathematical morphology. Biomedical Engineering, IEEE Transactions on, 40, 201-205.

Venes, D. Taber's cyclopedic medical dictionary, FA Davis.

Wagner, G., Freye, C., Palmeri, S., Roark, S., Stack, N., Harrell, F. \& Selvester, R. (1982) Evaluation of a QRS scoring system for estimating myocardial infarct size. I. Specificity and observer agreement. Circulation, 65, 342-347. 